\title{
THE NUMBER OF FACTORS IN A PAPERFOLDING SEQUENCE
}

\author{
Jean-Paul Allouche
}

We prove that the number of factors of length $k$ in any paperfolding sequence is equal to $4 k$ once $k \geqslant 7$.

\section{INTRODUCTION}

A factor of an infinite sequence $u=(u(n))_{n \geqslant 0}$ with values in $A$ is a word on $A$ occurring as $u(n) u(n+1) \cdots u(n+k-1)$ for some $n ; k$ is called the length of the factor.

The study of factors of infinite sequences goes back at least to Thue $[15,16]$ and has interested mathematicians and computer scientists working in combinatorics, symbolic dynamics, finitely generated groups, number theory, formal languages ... .

Among the questions which have been addressed is the problem of computing for a given finite sequence $u$ its complexity function $P_{u}$, where $P_{u}(k)$ is the number of factors of length $k$ in $u$. We quote here some results:

- if for some $k$ one has $P_{u}(k) \leqslant k$, then $u$ is ultimately periodic (see [13] for example),

- the sequences with minimal complexity which are not ultimately periodic satisfy $P_{u}(k)=k+1$; these are called Sturmian sequences (see [7] for example),

- if $u$ is an automatic sequence (in the sense of [5]), then one has $P_{u}(k) \leqslant$ $C k$ for some constant $C[6]$;

- if $u$ is the Thue-Morse sequence, then $P_{u}(k)$ has been computed $[4,10]$; it depends upon the digits of the binary expansion of $k$. More precisely the sequence $\left(P_{u}(k+1)-P_{u}(k)\right)_{k \geqslant 0}$ has only finitely many values and is an automatic sequence;

- if $u$ is an automatic sequence satisfying some technical requirements, then $\left(P_{u}(k+1)-P_{u}(k)\right)_{k \geqslant 0}$ is also automatic [14].

Received 28 June 1991

This paper was written while the author was visiting Macquarie University in Sydney. The author would like to thank warmly Jeff Shallit for numerous computations related to this problem and Alf van der Poorten for may enjoyable discussions.

Copyright Clearance Centre, Inc. Serial-fee code: 0004-9729/92 SA2.00+0.00. 
Very recently Shallit and the author proved in [2] that, for $u$ a generalised RudinShapiro sequence in the sense of $[1],(u$ counts the parity of the number of blocks $1 * \cdots * 1$ in the binary expansion of $n)$, the function $P_{u}(k)$ is ultimately affine. This result is somewhat surprising when compared to the complicated case of the Thue-Morse sequence.

We will prove here that the number of factors of length $k$ of any paperfolding sequence (see [8] for instance for a definition) is equal to $4 k$, provided $k \geqslant 7$. As a corollary we obtain that all generalised Rudin-Shapiro sequences in the sense of [12] (which except for the classical Rudin-Shapiro sequence are different from the sequences studied in [2]) have an ultimately affine complexity.

\section{A QUICK SURVEY OF PAPERFOLDING}

We recall that a paperfolding sequence is the sequence of ridges and valleys obtained by unfolding a sheet of paper which has been folded infinitely many times (see $[3,8$, $11,12]$ ). In other words the sequence $(u(n))_{n \geqslant 0}$ is a paperfolding sequence if and only if

$$
\begin{array}{rc}
u(4 n)=0 & (\text { respectively } 1) \\
u(4 n+2)=1 & (\text { respectively } 0) \\
u(2 n+1) & \text { is a paperfolding sequence. }
\end{array}
$$

Another way of generating these sequences is to view them as Toeplitz sequences [9]: given an infinite binary sequence $i=(i(n))_{n \geqslant 0}$ (sequence of "folding instructions"), one defines the paperfolding sequence $u_{i}=\left(u_{i}(n)\right)_{n \geqslant 0}$ with folding instructions $i$ by successively "filling holes":

- first step, one writes down the sequence $(i(0) \overline{i(0)})^{\infty}$ at the even places, which gives

$$
i(0) \bullet \overline{i(0)} \bullet i(0) \bullet \overline{i(0)} \bullet i(0) \bullet \overline{i(0)} \bullet \ldots
$$

- second step, one writes down the sequence $(i(1) \overline{i(0)})^{\infty}$ at the even holes, which gives

$$
i(0) i(1) \overline{i(0)} \bullet i(0) \overline{i(1)} \overline{i(0)} \bullet i(0) i(1) \overline{i(0)} \bullet \ldots
$$

and so on; the limit obtained after an infinite number of steps is the sequence $u_{i}$. 
Finally we mention the generation of a paperfolding sequence by "perturbed symmetry" (see [3]).

If $a$ is a letter ( 0 or 1 ), we define the operator $T_{a}$ by: for every word $M, T_{a}(M)=$ $M a \widetilde{M}$ (where $\widetilde{M}$ is obtained from $M$ by reading $M$ backwards, then replacing the 0 's by 1 's and the 1 's by 0 's).

Given a sequence $a_{0} a_{1} \cdots$, one then obtains a paperfolding sequence by starting, say, from 0 , and successively applying the operators $T_{a_{j}}$ :

$$
\begin{aligned}
T_{a_{0}}(0) & =0 a_{0} 1 \\
T_{a_{1}} T_{a_{0}}(0) & =0 a_{0} 1 a_{1} 0 \overline{a_{0}} 1 \\
T_{a_{2}} T_{a_{1}} T_{a_{0}}(0) & =0 a_{0} 1 a_{1} \overline{a_{0}} 1 a_{2} 0 a_{0} 1 \overline{a_{1}} 0 \overline{a_{0}} 1
\end{aligned}
$$

Note that the word obtained at each step is of length $2^{r}-1$ for some $r \geqslant 1$.

\section{O-FACTORS AND E-FACTORS IN A PAPERFolding SEQUENCE}

In the sequel we say that a factor of the paperfolding sequence $u_{i}=\left(u_{i}(n)\right)_{n \geqslant 0}$ (with folding instructions $i$ ) is an O-factor (respectively an E-factor) of $u_{i}$ if it occurs in $u_{i}$ as $u_{i}(n) u_{i}(n+1) \cdots u_{i}(n+k-1)$ with $n$ odd (respectively $n$ even). Note that a factor can simultaneously be an $\mathrm{O}$-factor and an E-factor (for instance the factor 0 and the factor 1 are simultaneously $O$-factors and E-factors of any paperfolding sequence).

We take a sequence of folding instructions beginning with $i(0)=\alpha, i(1)=\beta$, and write it as $\alpha \beta j$ (so $j$ is defined by $j(n)=i(n+2)$ ).

Applying the Toeplitz process twice, we obtain:

$$
\alpha \beta \bar{\alpha} \bullet \alpha \bar{\beta} \bar{\alpha} \bullet \alpha \beta \bar{\alpha} \bullet \alpha \bar{\beta} \bar{\alpha} \bullet \alpha \beta \bar{\alpha} \bullet \ldots
$$

We are now ready to state two lemmata:

LEMMA 1. The E-factors of length $\geqslant 4$ of a paperfolding sequence $u_{\alpha \beta_{j}}$ are in one of the following four disjoint classes:

$$
\begin{array}{ll}
\alpha \beta \bar{\alpha} \bullet & \ldots \\
\bar{\alpha} \bullet \alpha \bar{\beta} & \ldots \\
\alpha \bar{\beta} \bar{\alpha} \bullet & \ldots \\
\bar{\alpha} \bullet \alpha \beta & \ldots
\end{array}
$$


The $O$-factors of length $\geqslant 4$ of a paperfolding sequence $u_{\alpha \beta j}$ are in one of the following four disjoint classes:

$$
\begin{array}{ll}
\beta \bar{\alpha} \bullet \alpha & \ldots \\
\bullet \alpha \bar{\beta} \bar{\alpha} & \ldots \\
\bar{\beta} \bar{\alpha} \bullet \alpha & \ldots \\
\bullet \alpha \beta \bar{\alpha} & \ldots
\end{array}
$$

PROOF: Inspection of $\left({ }^{*}\right)$ reveals that the E-factors (respectively the O-factors) begin as written. Moreover the classes one obtains are disjoint regardless of $\alpha, \beta$ and the holes.

LEMMA 2. If a factor of a paperfolding sequence has length greater than or equal to 7 , then it cannot be simultaneously an o-factor and an $e$-factor.

PROOF: Once again inspecting $\left(^{*}\right)$ one sees that a factor of $u_{\alpha \beta j}$ of length greater than or equal to 7 begins in one of the following ways:

$$
\begin{array}{lll}
1- & \alpha \beta \bar{\alpha} \bullet \alpha \bar{\beta} \bar{\alpha} & \ldots \\
2- & \beta \bar{\alpha} \bullet \alpha \bar{\beta} \bar{\alpha} & \ldots \\
3- & \bar{\alpha} \bullet \alpha \bar{\beta} \bar{\alpha} \bullet \alpha & \ldots \\
4- & \bullet \bar{\beta} \bar{\alpha} \bullet \alpha \beta & \ldots \\
5- & \alpha \bar{\beta} \bar{\alpha} \bullet \alpha \beta \bar{\alpha} & \ldots \\
6- & \bar{\beta} \bar{\alpha} \bullet \alpha \beta \bar{\alpha} \bullet & \ldots \\
7- & \bar{\alpha} \bullet \alpha \beta \bar{\alpha} \bullet \alpha & \ldots \\
\text { 8- } & \bullet \alpha \beta \bar{\alpha} \bullet \alpha \bar{\beta} & \ldots
\end{array}
$$

The cited words of length 7 are different (whatever the values of $\alpha, \beta$, and the holes). In particular the E-factors (numbered 1, 3, 5, 7) and the $O$-factors (numbered $2,4,6,8)(!)$ are different.

\section{A ReCURRENCE RELATION FOR THE NUMBER OF O-Factors and E-Factors}

For $u_{i}=\left(u_{i}(n)\right)_{n \geqslant 0}$ a paperfolding sequence with folding instructions $i$, one defines:

$g_{i}(k)$ is the number of E-factors of length $k$ in $u_{i}$,

$h_{i}(k)$ is the number of O-factors of length $k$ in $u_{i}$.

How can we obtain an E-factor of length $4 k$ in $u_{\alpha \beta j}$ ? Once again inspecting $\left({ }^{*}\right)$ we see that an E-factor of length $4 k$ of $u_{\alpha \beta j}$ is of one of the following types:

1: $\quad \alpha \beta \bar{\alpha} \bullet \alpha \bar{\beta} \bar{\alpha} \bullet \alpha \beta \bar{\alpha} \bullet \quad \cdots$

2: $\bar{\alpha} \bullet \alpha \bar{\beta} \bar{\alpha} \bullet \alpha \beta \bar{\alpha} \bullet \alpha \bar{\beta} \quad \ldots$

3: $\alpha \bar{\beta} \bar{\alpha} \bullet \alpha \beta \bar{\alpha} \bullet \alpha \bar{\beta} \bar{\alpha} \bullet \quad \ldots$

4: $\bar{\alpha} \bullet \alpha \beta \bar{\alpha} \bullet \alpha \bar{\beta} \bar{\alpha} \bullet \alpha \beta \quad \ldots$ 
and these types are disjoint for $4 k \geqslant 4$ (Lemma 1). Moreover if one considers the subsequent steps in the Toeplitz process, one sees that the remaining holes are filled exactly by the E-factors of $u_{j}$ in Cases 1 and 2 and by the O-factors of $u_{j}$ in Cases 3 and 4.

Hence:

$$
\forall k \geqslant 1 \quad g_{\alpha \beta_{j}}(4 k)=2 g_{j}(k)+2 h_{j}(k) .
$$

In the same way we compute the quantities $g_{\alpha \beta j}(4 k+r)$ and $h_{\alpha \beta j}(4 k+r)$ for $r=$ $0,1,2,3$, obtaining the following proposition:

PROPOSITION. One has the following relations for $k \geqslant 1$ :

$$
\begin{aligned}
g_{\alpha \beta j}(4 k) & =2 g_{j}(k)+2 h_{j}(k), \\
h_{\alpha \beta j}(4 k) & =2 g_{j}(k)+2 h_{j}(k), \\
g_{\alpha \beta j}(4 k+1) & =2 g_{j}(k)+2 h_{j}(k), \\
h_{\alpha \beta j}(4 k+1) & =g_{j}(k)+g_{j}(k+1)+h_{j}(k)+h_{j}(k+1), \\
g_{\alpha \beta j}(4 k+2) & =g_{j}(k)+g_{j}(k+1)+h_{j}(k)+h_{j}(k+1), \\
h_{\alpha \beta j}(4 k+2) & =g_{j}(k)+g_{j}(k+1)+h_{j}(k)+h_{j}(k+1), \\
g_{\alpha \beta j}(4 k+3) & =g_{j}(k)+g_{j}(k+1)+h_{j}(k)+h_{j}(k+1), \\
h_{\alpha \beta j}(4 k+3) & =2 g_{j}(k+1)+2 h_{j}(k+1) .
\end{aligned}
$$

\section{Counting the FACTORS OF A PAPERFolding SEQUENCE}

We are now able to prove the theorem:

THEOREM . For any paperfolding sequence $u_{i}=\left(u_{i}(n)\right)_{n \geqslant 0}$, the number of factors of length $k, P_{u_{i}}(k)$, is given by:

$$
P_{u_{i}}(1)=2, P_{u_{i}}(2)=4, P_{u_{i}}(3)=8, P_{u_{i}}(4)=12, P_{u_{i}}(5)=18, P_{u_{i}}(6)=23 \text {, }
$$

and for all $k \geqslant 7, P_{u_{i}}(k)=4 k$.

Proof: Define

$$
V_{i}(k)=\left(\begin{array}{c}
g_{i}(k) \\
h_{i}(k) \\
g_{i}(k+1) \\
h_{i}(k+1)
\end{array}\right)
$$

The recurrence relations in the previous paragraph can be rewritten as:

$$
V_{\alpha \beta j}(4 k+4)=A_{r} V_{j}(k) \quad \forall k \geqslant 1
$$


where the matrices $A_{r}$ are given by

$$
\begin{array}{rlrl}
A_{0} & =\left(\begin{array}{llll}
2 & 2 & 0 & 0 \\
2 & 2 & 0 & 0 \\
2 & 2 & 0 & 0 \\
1 & 1 & 1 & 1
\end{array}\right), & A_{1}=\left(\begin{array}{llll}
2 & 2 & 0 & 0 \\
1 & 1 & 1 & 1 \\
1 & 1 & 1 & 1 \\
1 & 1 & 1 & 1
\end{array}\right), \\
A_{2}=\left(\begin{array}{llll}
1 & 1 & 1 & 1 \\
1 & 1 & 1 & 1 \\
1 & 1 & 1 & 1 \\
0 & 0 & 2 & 2
\end{array}\right), & A_{3}=\left(\begin{array}{llll}
1 & 1 & 1 & 1 \\
0 & 0 & 2 & 2 \\
0 & 0 & 2 & 2 \\
0 & 0 & 2 & 2
\end{array}\right) .
\end{array}
$$

We then notice (still using $\left({ }^{*}\right)$ and considering all possibilities for $\alpha, \beta$, and the holes) that, for every sequence of folding instructions $j$, one has:

$$
g_{j}(1)=h_{j}(1)=2, g_{j}(2)=h_{j}(2)=4, g_{j}(3)=4, h_{j}(3)=8, g_{j}(4)=h_{j}(4)=8 \text {. }
$$

Now we claim that for every sequence of instructions $j$, one has:

$$
\begin{aligned}
& \forall k \geqslant 1, k \text { even, } V_{j}(k)=\left(\begin{array}{c}
2 k \\
2 k \\
2 k \\
2 k+4
\end{array}\right), \\
& \forall k \geqslant 1, k \text { odd }, \quad V_{j}(k)=\left(\begin{array}{c}
2 k-2 \\
2 k+2 \\
2 k+2 \\
2 k+2
\end{array}\right) .
\end{aligned}
$$

The proof that this is true for every sequence $j$ and for every $k$ in $[1,4 n-1]$ follows easily by induction on $n$ and is left as an exercise for the reader.

Finally, using Lemma 2, we obtain that, for every sequence of instructions $i$, one has:

$$
\forall k \geqslant 7 \quad P_{u_{i}}(k)=g_{i}(k)+h_{i}(k)=4 k .
$$

The values of $P_{u_{i}}(k)$ for $1 \leqslant k \leqslant 6$ are computed by hand using $\left(^{*}\right)$ for a final time.

\section{THE NUMBER OF FACTORS OF THE GENERALISED RUdIN-SHAPIRO SEQUENCES}

(a) The sequences we consider here were introduced in [12] and are defined as follows: if $u_{i}$ is a paperfolding sequence, one defines $w_{i}$ by

$$
\begin{aligned}
& w_{i}(0)=0 \\
& w_{i}(n)=\sum_{t=0}^{n-1} u_{i}(t) \text { modulo } 2, \text { for } n \geqslant 1 .
\end{aligned}
$$


These sequences have the Rudin-Shapiro property that

$$
\left\|\sum_{n=0}^{N-1}(-1)^{w_{i}(n)} e^{2 i \pi n x}\right\|_{\infty} \leqslant C_{i} \sqrt{N} .
$$

We shall prove the following theorem (compare with the "other" generalised RudinShapiro sequences studied in [2]):

THEOREM 2. For any generalised Rudin-Shapiro sequence (in the sense of [12]) $w_{i}$ one has:

$$
\begin{aligned}
& P_{w_{i}}(1)=2, P_{w_{i}}(2)=4, P_{w_{i}}(3)=8, P_{w_{i}}(4)=16, P_{w_{i}}(5)=24, P_{w_{i}}(6)=36, \\
& P_{w_{i}}(7)=46,
\end{aligned}
$$

and for all $k \geqslant 8, P_{w_{i}}(k)=8 k-8$.

We first need a lemma:

LEMMA 3. Let $u_{i}=\left(u_{i}(n)\right)_{n \geqslant 0}$ be a paperfolding sequence. If $F=u_{i}(n) u_{i}(n+1)$ $\cdots u_{i}(n+k-1)$ is a factor of $u_{i}$ of length $k$, such that $\sum_{t=0}^{n-1} u_{i}(t)=a$, then there exists $n^{\prime}$ such that:

$$
\begin{array}{ll}
* \quad F=u_{i}\left(n^{\prime}\right) u_{i}\left(n^{\prime}+1\right) \cdots u_{i}\left(n^{\prime}+k-1\right) \\
* \quad \sum_{t=0}^{n^{\prime}-1} u_{i}(t)=1+a \text { modulo } 2 .
\end{array}
$$

Here we use the definition of paperfolding by means of "perturbed symmetry". If $M$ is a factor of $u_{i}$, then there exist two factors $X$ and $Y$ such that $X M Y$ is a left factor of $u_{i}$ (that is, beginning at place 0 ) of length $2^{s}-1$ for some $s \geqslant 1$.

Now applying perturbed symmetry operators three times we see that $u_{i}$ begins with:

\section{$X M Y \alpha \tilde{Y} \widetilde{M} \tilde{X} \beta X M Y \bar{\alpha} \widetilde{Y} \widetilde{M} \widetilde{X} \gamma X M Y \alpha \widetilde{Y} \widetilde{M} \widetilde{X} \bar{\beta} X M Y \bar{\alpha} \tilde{Y} \widetilde{M} \tilde{X}$} ( $\bar{\alpha}$ means $1+\alpha$ modulo 2 ).

$M$ occurs four times. Denoting by $s(X)$ the sum modulo 2 of the letters of $X$ one sees that:

* the first occurrence of $M$ is preceded by a word of sum $s(X)$,

* the second occurrence of $M$ is preceded by a word of sum $s(X)+$ $s(X M Y)+s(\tilde{Y} \widetilde{M} \tilde{X})+\alpha+\beta ;$ but for every word $Z, s(Z)+s(\tilde{Z})=$ length of $Z$ modulo 2 ; hence this sum is equal to $s(X)+1+\alpha+\beta$;

* the third occurrence of $M$ is preceded by a word of sum $s(X)+1+\alpha+$ $\beta+$ length $(X M Y)+\bar{\alpha}+\gamma=s(X)+1+\beta+\gamma$;

* the fourth occurrence of $M$ is preceded by a word of sum $s(X)+1+\beta+$ $\gamma+\operatorname{length}(X M Y)+\alpha+\bar{\beta}=s(X)+1+\alpha+\gamma$. 
As one cannot simultaneously have

$$
1+\alpha+\beta=1+\beta+\gamma=1+\alpha+\gamma=0 .
$$

one of these sums is equal to 1 , proving the lemma.

Proof of Theorem 2: (b) Let $u_{i}=\left(u_{i}(n)\right)_{n \geqslant 0}$ be a paperfolding sequence with sequence of folding instructions $i$, and let $w_{i}$ be defined by:

$$
\begin{aligned}
w_{i}(0) & =0 \\
w_{i}(n) & =\sum_{t=0}^{n-1} u_{i}(t) \quad \text { for } \quad n \geqslant 1 .
\end{aligned}
$$

Let $F_{u_{i}}(k)$ be the set of factors of $u_{i}$ of length $k$ and similarly define $F_{w_{i}}(k)$. Now define on $F_{w_{i}}(k)(k \geqslant 2)$ the map $\psi_{k}$ by

$$
\psi_{k}\left(e_{0}, e_{1}, \cdots, e_{k-1}\right)=\left(e_{0}, e_{0}+e_{1}, \cdots, e_{k-2}+e_{k-1}\right)
$$

(the sums being taken modulo 2).

If $n$ is such that $w_{\mathbf{i}}(n+t)=e_{t}$, for $0 \leqslant t \leqslant k-1$, we see that $u_{i}(n+t)=$ $w_{i}(n+t)+w_{i}(n+t+1)=e_{t}+e_{t+1}$ for $0 \leqslant t \leqslant k-2$, hence $\psi_{k}$ maps $F_{w_{i}}(k)$ to $\{0,1\} \times F_{u_{i}}(k-1)$.

Clearly $\psi_{k}$ is one-to-one. To see that $\psi_{k}$ is onto, we note that given $\left(a_{0}, a_{1}, \cdots, a_{k-1}\right)$ in $\{0,1\} \times F_{u_{i}}(k-1)$, there exists $n$ such that $u_{i}(n+t)=a_{t+1}$ for $0 \leqslant t \leqslant k-2$.

Hence:

$$
\begin{aligned}
w_{i}(n+1) & =w_{i}(n)+a_{1} \\
w_{i}(n+2) & =w_{i}(n)+a_{1}+a_{2} \\
\cdots & \\
w_{i}(n+k-1) & =w_{i}(n)+a_{1}+a_{2}+\cdots+a_{k-1} .
\end{aligned}
$$

So if $w_{i}(n)=a_{0}$, then $w_{i}(n+t)=a_{0}+a_{1}+a_{2}+\cdots+a_{t}$ for every $t$ in $[0, k-1]$, and this gives an element in $F_{w_{i}}(k)$ such that

$$
\psi_{k}\left(w_{i}(n), w_{i}(n+1), \cdots, w_{i}(n+k-1)\right)=\left(a_{0}, a_{1}, \cdots, a_{k-1}\right) .
$$

If now $w_{i}(n)=1+a_{0}$, (that is $\left.\sum_{t=0}^{n-1} u_{i}(t)=1+a_{0}\right)$, then by Lemma 3 there exists an integer $n^{\prime}$ such that $\sum_{t=0}^{n^{\prime}-1} u_{i}(t)=a_{0}$, that is, $w_{i}\left(n^{\prime}\right)=a_{0}$ and $u_{i}\left(n^{\prime}+t\right)=u_{i}(n+t)=$ $a_{t+1}$ for $0 \leqslant t \leqslant k-2$. 
Hence $w_{i}\left(n^{\prime}+t\right)=a_{0}+a_{1}+\cdots+a_{t}$ for $0 \leqslant t \leqslant k-1$, and

$$
\psi_{k}\left(w_{i}\left(n^{\prime}\right), w_{i}\left(n^{\prime}+1\right), \cdots, w_{i}\left(n^{\prime}+k-1\right)\right)=\left(a_{0}, a_{1}, \cdots, a_{k-1}\right) .
$$

Thus finally $\psi_{k}$ is a bijection from $F_{w_{i}}(k)$ onto $\{0,1\} \times F_{u_{i}}(k-1)$, which proves that:

$$
P_{w_{i}}(k)=2 P_{u_{i}}(k-1), \quad \text { for } k \geqslant 2 \text {, }
$$

and our Theorem 2 is now nothing but a reformulation of Theorem 1 .

\section{REFERENCES}

[1] J.-P. Allouche and P. Liardet, 'Generalized Rudin-Shapiro sequences', Acta. Arith. 60 (1991), 1-27.

[2] J.-P. Allouche and J. Shallit, 'Suites à complexité ultimement affine', Congrès "Thémate", Luminy (1991).

[3] A. Blanchard and M. Mendès France, 'Symétrie et transcendance', Bull. Sci. Math. 106 (1982), 325-335.

[4] S. Brlek, 'Enumeration of factors in the Thue-Morse word', Discrete Appl. Math. 24 (1989), 83-96.

[5] G. Christol, T. Kamae, M. Medès France and G. Rauzy, 'Suites algébriques, automates et substitutions', Bull. Soc. Math. France 108 (1980), 401-419.

[6] A. Cobham, 'Uniform tag sequences', Math. Systems Theory 6 (1972), 164-192.

[7] E.M. Coven and G.A. Hedlund, 'Sequences with minimal block growth', Math. Systems Theory 7 (1973), 138-153.

[8] M. Dekking, M. Medès France and A. van der Poorten, 'FOLDS!', Math. Intelligencer 4 (1982), 130-138; 173-181; 190-195.

[9] K. Jacobs and M. Keane, '0-1-sequences of Toeplitz type', Z. Wahrsch. verw. Geb. 13 (1969), 123-131.

[10] A. de Luca and S. Varricchio, 'Some combinatorial properties of the Thue-Morse sequence', Theoret. Comput. Sci. 63 (1989), 333-348.

[11] M. Mendès France and A.J. van der Poorten, 'Arithmetic and analytic properties of paperfolding sequences', (dedicated to K. Mahler), Bull. Austral. Math. Soc. 24 (1981), 123-131.

[12] M. Mendès France and G. Tenenbaum, 'Dimension des courbes planes, papiers pliés et suites de Rudin-Shapiro', Bull. Soc. Math. France 109 (1981), 207-215.

[13] M. Morse and G.A. Hedlund, 'Symbolic dynamics', Amer. J. Math. 60 (1938), 815-866.

[14] T. Tapsoba, Complexité de suites automatiques, Thèse de troisième cycle II (Université d'Aix - Marseille, 1987).

[15] A. Thue, 'Über unendliche Zeichenreihen', Norske vid. Selsk. Skr., I. Mat. Nat. Kl., Christiana 7 (1906), 1-22.

[16] A. Thue, 'Über die gegenseitige Lage gleicher Teile gewisser Zeichenreihen', Vorske vid. Selsk. Skr., I. Mat. Nat. Kl., Christiana 1 (1912), 1-67. 
C.N.R.S. U.R.A 0226

Mathématiques et Informatique

351, cours de la Libération

F-33405 Talence, Cedex

France 